\title{
Rechtsprechung
}

\section{Zu einem Rechtshilfeersuchen über die Herausgabe illegal erlangter Kulturgüter} Tribunale federale, Urteil vom 12. November 2007 - [T 0/2] 1 A.47/2007

\begin{abstract}
Es reicht aus, wenn die Angaben in einem Rechtshilfeersuchen über die Herausgabe deliktisch erworbener Kulturgüter die Prüfung ermöglichen, ob ausreichende Anhaltspunkte für eine rechtshilfefähige Straftat vorliegen, ob Verweigerungsgründe gegeben sind bzw. in welchem Umfang dem Begehren entsprochen werden muss.

Dass eine ersuchende Behörde die Herausgabe der Gegenstände nicht nur zu Beweis-, sondern auch zu Einziehungszwecken beantragt hätte, stellt kein Rechtshilfehindernis dar. Die weitere Verwendung der herausgegebenen Gegenstände für eine strafrechtliche Einziehung müsste von den ersuchenden Behörden jedoch separat beantragt und von den schweizerischen Rechtshilfebehörden ausdrücklich bewilligt werden. (Leitsätze d. Redaktion)
\end{abstract}

\section{$[\ldots]$}

\section{Sachverhalt:}

- A. Die italienische Strafustiz führt eine Strafuntersuchung gegen A.X. und Mitbeteiligte wegen illegalen Kulturgütertransfers, Hehlerei, Nichtanmeldung von archäologischen Funden und Zugehörigkeit zu einer kriminellen Vereinigung. Mit Schreiben vom 12. Juli 2000 ersuchte die römische Staatsanwaltschaft die schweizerischen Behörden um internationale Rechtshilfe. Das Ersuchen wurde mehrfach ergänzt. Mit Eintretens- und Zwischenverfügung vom 1. Oktober 2001 ordnete die Staatsanwaltschaft Basel-Stadt in mehreren Liegenschaften Hausdurchsuchungen und Beschlagnahmungen an.

B. Gegen B.X., die Ehefrau von A.X., eröffnete die Basler Staatsanwaltschaft am 24. Oktober 2001 ein separates Strafverfahren wegen des Verdachtes der Hehlerei, des Betruges, der Urkundenfälschung und weiterer Delikte. In diesem Zusammenhang stellten die kantonalen Behörden am 7. Oktober 2002 ihrerseits ein Ersuchen um Rechtshilfe, welches von den italienischen Behörden am 21. Dezember 2003 beantwortet wurde.

C. Am 12. November 2002 erließ die Staatsanwaltschaft eine Teil-Schlussverfügung betreffend rechtshilfeweise Herausgabe von Kopien von beschlagnahmten Geschäftsunterlagen. Nachdem das Bundesgericht dagegen erhobene Beschwerden mit Urteilen vom 16. Juli 2004 (Verfahren 1A.59/2004) bzw. 9. Mai 2005 (Verfahren 1A.37/2005) abwies, ist die betreffende partielle Schlussverfügung in Rechtskraft erwachsen.

D. Mit Schlussverfügung vom 22. Februar 2006 bewilligte die Staatsanwaltschaft die rechtshilfeweise Herausgabe von diversen beschlagnahmten Kunst- und Kulturgegenständen sowie von weiteren Unterlagen (Rechtshilfeakten sowie sichergestellte Photographien von Kunst- und Kulturgegenständen). Die Herausgabe wurde (gestützt auf Art. 74 IRSG) zu Beweiszwecken verfügt. Einen von A.X. gegen die Schlussverfügung erhobenen Rekurs wies das Strafgericht Basel-Stadt, Rekurskammer, mit Entscheid vom 2. April 2007 ab, soweit es darauf eintrat.
E. Gegen den Entscheid des Strafgerichtes gelangte A.X. mit Verwaltungsgerichtsbeschwerde vom 14. Mai 2007 an das Bundesgericht. Er beantragt zur Hauptsache die Aufhebung des angefochtenen Entscheides und die Verweigerung der Rechtshilfe. Die kantonalen Behörden und das Bundesamt für Justiz beantragen je die Abweisung der Beschwerde. [...]

\section{Das Bundesgericht zieht in Erwägung:}

1. Die streitige Schlussverfügung datiert vom 22. Februar 2006. Damit sind hier in prozessualer Hinsicht die altrechtlichen Vorschriften (Art. 97 ff. OG i.V.m. aArt. 25 und aArt. 80e ff. IRSG) anwendbar (Art. 110b IRSG; vgl. BGE 133 IV 58 E. 1.1 S. 60).

2. Für die Rechtshilfe zwischen der Schweiz und Italien sind in erster Linie die Bestimmungen des Europäischen Übereinkommens über die Rechtshilfe in Strafsachen vom 20. April 1959 (EUeR, SR 0.351.1) maßgebend, dem sowohl die Schweiz als auch Italien beigetreten sind. Soweit dieser Staatsvertrag bestimmte Fragen nicht abschließend regelt, gelangt das schweizerische Landesrecht, d.h. das Bundesgesetz vom 20. März 1981 über internationale Rechtshilfe in Strafsachen (IRSG, SR 351.1) und die diesbezügliche Verordnung vom 24. Februar 1982 (IRSV, SR 351.11), zur Anwendung. Im vorliegenden Fall ist ferner auf das UNESCO-Kulturgütertransfer-Abkommen vom 14. November 1970 hinzuweisen (SR 0.444.1, in Kraft getreten für die Schweiz am 3. Januar 2004, für Italien am 2. Januar 1979) sowie auf das Bundesgesetz über den internationalen Kulturgütertransfer vom 20. Juni 2003 (Kulturgütertransfergesetz, KGTG [SR 444.1], in Kraft seit 1. Juni 2005; insbes. Art. 22-29 KGTG). [...]

2.2. Zulässige Beschwerdegründe sind die Verletzung von Bundesrecht (inklusive Staatsvertragsrecht), einschließlich Überschreitung oder Missbrauch des Ermessens, sowie die unzulässige oder offensichtlich unrichtige Anwendung ausländischen Rechts in den Fällen nach Art. 65 IRSG (aArt. 80i Abs. 1 IRSG). [...]

2.3. Das Bundesgericht ist an die Begehren der Parteien nicht gebunden (aArt. 25 Abs. 6 IRSG). Im Rahmen der Verwaltungsge- 
richtsbeschwerde prüft es jedoch grundsätzlich nur Rechtshilfevoraussetzungen, die bestritten werden (BGE 132 II 81 E. 1.4 S. 84; 130 || 337 E. 1.4 S. 341; 123 || 134 E. 1d S. 136 f.). [...]

4. In der Sache macht der Beschwerdeführer geltend, der Tatverdacht gegen inn habe sich nicht ausreichend erhärtet und das Rechtshilfeerfordernis der beidseitigen Strafbarkeit sei nicht erfüllt. Bis zum Inkrafttreten des Bundesgesetzes über den internationalen Kulturgütertransfer (am 1. Juni 2005) habe nach schweizerischem Recht „,keine spezielle Strafbarkeit für den Handel mit Kulturgütern“ bestanden.

4.1. Die Vertragsparteien des EUeR können sich das Recht vorbehalten, die Erledigung von Ersuchen um Durchsuchung oder Beschlagnahme der Bedingung zu unterwerfen, dass die dem Ersuchen zugrunde liegende strafbare Handlung sowohl nach dem Recht des ersuchenden als auch nach dem des ersuchten Staates strafbar ist (Art. 5 Ziff. 1 lit. a EUeR). Die Schweiz hat für die Durchführung prozessualer Zwangsmassnahmen eine entsprechende Erklärung angebracht. Art. 64 Abs. 1 IRSG bestimmt (für die akzessorische Rechtshilfe), dass prozessuale Zwangsmassnahmen nur angewendet werden dürfen, wenn aus der Darstellung des Sachverhalts im Ersuchen hervorgeht, dass die im Ausland verfolgte Handlung die objektiven Merkmale eines nach schweizerischem Recht strafbaren Tatbestandes aufweist. Der Rechtshilferichter prüft, ob der im Ausland verübte inkriminierte Sachverhalt, sofern er - analog - in der Schweiz begangen worden wäre, die Tatbestandsmerkmale einer schweizerischen Strafnorm erfüllen würde (vgl. BGE 132 II 81 E. 2.7.2 S. 90 f.; 129 II 462 E. 4.4 S. 465; 118 lb 543 E. 3b/aa S. 546).

Das Ersuchen hat die mutmaßliche strafbare Handlung zu bezeichnen und eine kurze Darstellung des Sachverhaltes zu enthalten. Die Bewilligung internationaler Rechtshilfe setzt voraus, dass sich aus der Sachdarstellung des Ersuchens hinreichende Verdachtsmomente für den untersuchten deliktischen Vorwurf ergeben (vgl. Art. 14 Ziff. 1-2 EUeR). Von den Behörden des ersuchenden Staates kann jedoch nicht verlangt werden, dass sie den Sachverhalt, der Gegenstand des hängigen Strafverfahrens bildet, lückenlos und völlig widerspruchsfrei darstellen. Das wäre mit dem Sinn und Zweck des Rechtshilfeverfahrens unvereinbar, ersucht doch ein Staat einen andern gerade deswegen um Unterstützung, damit er die bisher im Dunkeln gebliebenen Punkte klären kann. Es reicht daher - unter dem Gesichtspunkt des hier maßgebenden EUeR - aus, wenn die Angaben im Rechtshilfeersuchen den schweizerischen Behörden ermöglichen zu prüfen, ob ausreichende Anhaltspunkte für eine rechtshilfefähige Straftat vorliegen, ob Verweigerungsgründe gegeben sind bzw. in welchem Umfang dem Begehren allenfalls entsprochen werden muss. Es kann auch nicht verlangt werden, dass die ersuchende Behörde die Tatvorwürfe bereits abschließend mit Beweisen belegt. Der Rechtshilferichter hat weder Tat- noch Schuldfragen zu prüfen und grundsätzlich auch keine Beweiswürdigung vorzunehmen, sondern ist vielmehr an die Sachdarstellung im Ersuchen gebunden, soweit sie nicht durch offensichtliche Fehler, Lücken oder Widersprüche sofort entkräftet wird (vgl. BGE 132 II $81 \mathrm{E}$. 2.1 S. 85 mit Hinweisen).

4.2. Die Sachdarstellung des Ersuchens sowie die vorläufigen Ergebnisse der italienischen Strafuntersuchung bzw. der in der Schweiz rechtshilfeweise erfolgten Beweismaßnahmen werden im angefochtenen Entscheid wie folgt zusammengefasst: Die Ehefrau des Beschwerdeführers habe (namentlich in den staatsanwaltlichen Einvernahmen vom 25. und 28. Oktober 2001) eingeräumt, dass ein Großteil der beschlagnahmten Kulturgüter in Italien illegal ausgegraben und ohne Papiere an sie verkauft worden sei. In der Folge seien gefälschte Unterlagen erstellt worden, mit denen vorgetäuscht werden sollte, dass die Gegenstände schon seit Jahren in ihrem Besitze gewesen wären.

Im Dezember 2005 habe die ersuchende Behörde einen Bericht über die bisherigen Ermittlungsergebnisse vorgelegt. Dieser enthalte unter anderem ein Kurzgutachten vom 19. Oktober 2005 über die vorläufige Auswertung der bereits an Italien übermittelten Geschäftsunterlagen. Darin würden die Verflechtungen des Beschwerdeführers und seiner Ehefrau aufgezeigt mit Personen, die ebenfalls des illegalen Handels mit archäologischen Gegenständen angeschuldigt seien. Die ersuchende Behörde lege nachvollziehbar die Modalitäten dar, nach denen deliktisch erworbene Kulturgüter in den legalen Kunst- und Antikenmarkt eingeschleust worden seien. Teilweise werde der illegale Kulturgütertransfer bereits im Einzelnen belegt. Im Kurzgutachten werde unter anderem dokumentiert, dass zwei im Lager der Ehefrau des Beschwerdeführers beschlagnahmte Marmorskulpturen (Beschlagnahmungen Nrn. 1427 und 1444) im Jahre 1988 aus dem städtischen Museum von Terracina gestohlen worden seien. In einem einschlägigen Urteil eines römischen Strafgerichtes vom 13. Dezember 2004, gemäß dem ein Mittäter zu zehn Jahren Freiheitsstrafe verurteilt worden sei, werde der Beschwerdeführer ausdrücklich als Beteiligter erwähnt.

4.3. Der Beschwerdeführer legt keine offensichtlichen Fehler oder Widersprüche dar, welche die genannten konkreten Verdachtsgründe ohne weiteres dahinfallen ließen. [...]

4.4. Es kann offen bleiben, ob im vorliegenden Fall für die Frage der beidseitigen Strafbarkeit auf das (am 1. Juni 2005 in Kraft gesetzte) eidgenössische Kulturgütertransfergesetz abgestellt werden kann. Dafür spräche, dass die streitige Schlussverfügung vom 22. Februar 2006 datiert. Aber selbst wenn die Strafbestimmungen von Art. 24-29 KGTG bei der Prüfung der beidseitigen Strafbarkeit nicht zu berücksichtigen wären, fiele der von der ersuchenden Behörde dargelegte Sachverhalt im Falle einer strafrechtlichen Verurteilung namentlich unter die Straftatbestände der Hehlerei (Art. 160 StGB) und der Urkundenfälschung (Art. 251 StGB; vgl. auch schon das konnexe frühere Urteil des Bundesgerichtes 1A.59/2004 vom 16. Juli 2004, E. 6.1, S. 12 f.). Wie im Ersuchen und dessen Beilagen erwähnt wird, ist der inkriminierte Sachverhalt auch nach italienischem Recht strafbar. Damit ist das Rechtshilfeerfordernis der beidseitigen Strafbarkeit (Art. 5 Ziff. 1 lit. a EUeR) erfüllt. [...] 
4.5. Der Beschwerdeführer rügt im genannten Zusammenhang eine Verletzung des rechtlichen Gehörs, da das Strafgericht seinen Antrag auf Einholung eines archäologischen Gutachtens (zur Herkunftsbestimmung der beschlagnahmten Gegenstände) zu Unrecht abgewiesen habe. Die Rüge erweist sich als unbegründet. Das Rechtshilfeverfahren ist kein Strafprozess. Es ist Aufgabe der Strafjustizbehörden des ersuchenden Staates, die notwendigen Beweise zu erheben. Wie dargelegt, hat der Rechtshilferichter zur Frage der Strafbarkeit weder Tat- noch Schuldgesichtspunkte zu beurteilen und grundsätzlich auch keine Beweiswürdigung vorzunehmen. Er ist vielmehr an die Sachdarstellung im Ersuchen gebunden, soweit sie nicht durch offensichtliche Fehler, Lücken oder Widersprüche sofort entkräftet wird (vgl. oben, E. 4.1). Es ist daher Sache der zuständigen italienischen Untersuchungsbehörde, die Behauptungen des Beschwerdeführers zur Herkunft der beschlagnahmten Gegenstände zu prüfen und nötigenfalls entsprechende Beweismaßnahmen zu treffen. [...]

5. Weiter wird gerügt, durch die Herausgabe der betroffenen Gegenstände erhielten die italienischen Untersuchungsbehörden keine zusätzlichen relevanten Informationen. Außerdem sei es Sache der Rechtshilfebehörde, eine Aussonderung der beschlagnahmten Gegenstände im Hinblick auf deren mögliche Beweisrelevanz vorzunehmen. Die kantonalen Behörden seien dabei nicht nach den in BGE 130 II 17 f. dargelegten Regeln vorgegangen.

5.1. Gemäß Art. 14 Ziff. 1 lit. b EUeR muss die ersuchende Behörde den Gegenstand und den Grund ihres Gesuches spezifizieren. Daraus leitet die Praxis ein Verbot der Beweisausforschung ab. Dieses richtet sich gegen Beweisaufnahmen „auf's Geratewohl". Es dürfen keine strafprozessualen Untersuchungshandlungen zur Auffindung von Belastungsmaterial zwecks nachträglicher Begründung eines Tatverdachtes (oder zur Verfolgung nicht rechtshilfefähiger Delikte) durchgeführt werden. Eine hinreichend präzise Umschreibung der Verdachtsgründe soll möglichen Missbräuchen vorbeugen. Es sind grundsätzlich (unter Vorbehalt von Art. 6 EUeR bzw. Art. 74-74a IRSG, vgl. dazu unten, E. 6) alle gewünschten Gegenstände zu übermitteln, welche sich auf den im Ersuchen dargelegten Verdacht beziehen können. Mithin muss eine ausreichende inhaltliche Konnexität zwischen dem untersuchten Sachverhalt und den fraglichen Objekten erstellt sein (vgl. BGE 129 || 462 E. 5.3 S. 467 f.; 122 || 367 E. 2c S. 371; 121 II 241 E. 3a S. 242 f., je mit Hinweisen). Bei der Frage, welche Rechtshilfemaßnahmen geboten und zulässig erscheinen, ist außerdem das Ersuchen sachgerecht zu interpretieren. Damit können namentlich unnötige Prozessleerläufe (durch das Einreichen neuer konnexer Ersuchen) vermieden werden (BGE 121 II 241 E. 3a S. 243).

Es ist Aufgabe der ersuchten Rechtshilfebehörde, diejenigen Gegenstände auszuscheiden, für die keine Rechtshilfe zulässig ist. Daher muss die ersuchte Behörde grundsätzlich aufzeigen, dass zwischen den von der Rechtshilfe betroffenen Objekten und dem Gegenstand der Strafuntersuchung ein ausreichender Sachzusammenhang besteht (vgl. BGE 130 II 14 E. 4.3 S. 16 f.; 122 II 367 E. 2c S. 371). Im Verfahren der Verwaltungsgerichtsbeschwer- de forscht das Bundesgericht jedoch nicht von sich aus nach Beweismitteln, die im ausländischen Verfahren (mit Sicherheit) nicht erheblich sein könnten. Es obliegt dem Betroffenen, schon im Rechtshilfeverfahren gegenüber der ausführenden Behörde konkret darzulegen, welche einzelnen Gegenstände für die Strafuntersuchung offensichtlich entbehrlich seien, und diese Auffassung auch zu begründen (vgl. BGE 130 II 14 E. 4.3-4.4 S. 17 f.; 126 II 258 E. 9b/aa S. 262; 122 II 367 E. 2d S. 372, je mit Hinweisen).

5.2. Im angefochtenen Entscheid wird erwogen, die Staatsanwaltschaft habe nicht etwa die Herausgabe aller beschlagnahmten Objekte zu Beweiszwecken an die ersuchende Behörde verfügt. Vielmehr sei differenziert und sorgfältig, gestützt auf die Berichte der italienischen Sachverständigen, eine Aussonderung erfolgt. Dabei seien nur diejenigen Gegenstände erfasst worden, welche mit größter Wahrscheinlichkeit italienischer Herkunft seien und unter die verdachtsbegründenden Sachdarlegungen des Ersuchens fielen. Es liege auf der Hand, dass eine Herausgabe zu weiteren Untersuchungs- und Beweiszwecken sachlich geboten sei. Neben einer sorgfältigen archäologischen Herkunftsbestimmung drängten sich spurentechnische Untersuchungen der Gegenstände auf. Eine bloße Analyse von photographischen Aufnahmen reiche dafür nicht aus. Einzelne archäologische Fundstücke wiesen Erdanhaftungen auf. Teils seien sie eingepackt, teils nach ihrer Ausgrabung offenbar absichtlich zerschlagen und wieder zusammengesetzt worden. Aus den entsprechenden Anhaltspunkten könnten Fachleute Rückschlüsse ziehen über die näheren Umstände des Fundes, Fundort, Fundzeit etc. Insofern seien die herauszugebenden Gegenstände beweisgeeignet und beweisrelevant. Eine Expertisierung durch italienische Sachverständige in der Schweiz sei im vorliegenden Fall (mit tausenden zu begutachtenden Objekten) zu aufwändig und zu kompliziert. Sie würde insbesondere eine Verlagerung der nötigen technischen, wissenschaftlichen und personellen Infrastruktur in die Schweiz voraussetzen.

5.3. Wie sich aus den Akten ergibt, hat die Staatsanwaltschaft eine Aussonderung im Sinne der dargelegten Praxis vorgenommen. Zuvor (sowie anschließend im Rekursverfahren vor Strafgericht) erhielten die Betroffenen auch Gelegenheit, sich zur Beweisrelevanz der beschlagnahmten Gegenstände zu äußern. Im Gegensatz zu seiner Ehefrau hat sich der Beschwerdeführer jedoch (soweit aus den umfangreichen Akten ersichtlich) dazu nicht vernehmen lassen. In seiner Verwaltungsgerichtsbeschwerde bestreitet er zwar pauschal die Beweistauglichkeit der ausgesonderten Gegenstände. Er setzt sich jedoch weder mit den stichhaltigen Einwänden des angefochtenen Entscheides auseinander, noch legt er dar, bei welchen konkreten Gegenständen eine Sachkonnexität bzw. Erheblichkeit für die hängige Strafuntersuchung offensichtlich entfiele. Die Frage, ob im vorliegenden Fall eine Begutachtung in der Schweiz genüge oder gar vorzuziehen sei, wird im angefochtenen Entscheid mit sachlich überzeugenden Argumenten verneint (vgl. dazu oben, E. 5.2, sowie unten, E. 6.3). Auch das vom Beschwerdeführer geltend gemachte Transportrisiko stellt hier kein Rechtshilfehindernis dar, zumal gemäß den vorliegenden Akten bereits im Sommer 
2005 ein fachmännischer sorgfältiger Transport der beschlagnahmten Objekte in neue Lagerräumlichkeiten erfolgt ist und dem kantonalen Rechtshilferichter in diesem Zusammenhang ein weiter Ermessensspielraum zusteht (vgl. BGE 123 II 268 E. 4a S. 274 , E. 5 S. 278)

6. Schließlich rügt der Beschwerdeführer, die Herausgabe von Wertgegenständen führe im vorliegenden Fall zu einer bundesrechtswidrigen Umgehung von Art. 74a IRSG. Zwar hätten die italienischen Behörden eventualiter die Herausgabe zu bloßen Beweiszwecken beantragt. Der "wirkliche Beweggrund" des Ersuchens sei jedoch die definitive strafrechtliche Einziehung der Gegenstände zugunsten des italienischen Fiskus. Es sei davon auszugehen, dass die Vermögenswerte, sobald sie in die Verfügungsmacht der italienischen Behörden geraten, nicht mehr herausgegeben würden.

6.1. Die Übermittlung von Beweisstücken wird in Art. 3 Ziff. 1 EUeR ausdrücklich als zulässige Rechtshilfemaßnahme erwähnt. Die Gegenstände, die in Erledigung eines Rechtshilfeersuchens übermittelt wurden, sind vom ersuchenden Staat allerdings so bald wie möglich an den ersuchten Staat zurückzugeben, sofern dieser nicht darauf verzichtet (Art. 6 Ziff. 2 EUeR). Der ersuchte Staat kann die Übergabe von Gegenständen, um deren Übermittlung ersucht worden ist, auch aufschieben, wenn er sie für ein anhängiges Strafverfahren benötigt (Art. 6 Ziff. 1 EUeR). Jede Verweigerung von Rechtshilfe ist im Übrigen zu begründen (Art. 19 EUeR).

6.2. Das IRSG ist anwendbar, soweit das EUeR keine abschlieBende Regelung enthält bzw. wenn sich (nach dem so genannten "Günstigkeitsprinzip") aus dem IRSG eine weitergehende Rechtshilfe ergibt (BGE 129 || 462 E. 1.1 S. 464; vgl. auch BGE 132 II 81 E. 1.1 S. 83). Das eidgenössische Recht sieht vor, dass Gegenstände oder Vermögenswerte, die zu Beweiszwecken rechtshilfeweise beschlagnahmt wurden, der zuständigen ausländischen Behörde auf deren Ersuchen hin und gestützt auf eine Schlussverfügung (gemäß Art. 80d IRSG) zur Verfügung gestellt werden (Art. 74 Abs. 1 IRSG). Macht ein Dritter, der gutgläubig Rechte erworben hat (oder eine Behörde bzw. der Geschädigte), Rechte an den zu Beweiszwecken herauszugebenden Gegenständen oder Vermögenswerten geltend, werden diese nur übermittelt, wenn der ersuchende Staat deren kostenlose Rückgabe nach Abschluss seines Verfahrens zusichert (Art. 74 Abs. 2 IRSG). Die Herausgabe zu Beweiszwecken kann außerdem aufgeschoben werden, solange die Gegenstände oder Vermögenswerte für ein in der Schweiz hängiges Strafverfahren benötigt werden (Art. 74 Abs. 3 IRSG). Dies kann insbesondere in Frage kommen, wenn das schweizerische Strafverfahren schon weit vorangeschritten ist oder der Deliktsschwerpunkt in der Schweiz liegt (vgl. BGE 123 II 268 E. 5 S. 278).

Eine rechtshilfeweise Herausgabe zur Einziehung oder Rückerstattung an den Berechtigten durch den ersuchenden Staat wäre nur unter den Voraussetzungen von Art. 74a IRSG zulässig. Insbesondere erfolgt eine Herausgabe zu solchen Zwecken grundsätzlich nur gestützt auf einen rechtskräftigen und vollstreckbaren gerichtlichen Einziehungsentscheid des ersuchen- den Staates (Art. 74a Abs. 3 IRSG; vgl. BGE 131 II 169 E. 6 S. 175; 123 II 268 E. 4a S. 274, E. 4b/aa S. 275; 595 E. 4e-f S. 604-606, E. 5e S. $611 \mathrm{f}$., je mit Hinweisen).

6.3. Im angefochtenen Entscheid wird erwogen, die Schlussverfügung basiere auf Art. 74 IRSG, d.h. die Herausgabe der ausgesonderten Gegenstände an die ersuchende Behörde erfolge „ausschließlich zu Beweiszwecken“. Die Voraussetzungen für eine definitive Herausgabe zur strafrechtlichen Einziehung (nach Art. 74a IRSG) seien hingegen im vorliegenden Fall offensichtlich nicht erfüllt. Angesichts der bisherigen Ermittlungsergebnisse bestehe kein Zweifel daran, dass ein Grossteil des beschlagnahmten und photographisch dokumentierten Materials aus Italien stamme. Für eine abschließende Beurteilung und Zuordnung der einzelnen Gegenstände sei jedoch eine direkte, physische archäologische Untersuchung im Rahmen der in Italien hängigen Strafuntersuchung erforderlich. Neben einer kunsthistorisch-archäologischen Begutachtung beabsichtige die italienische Strafjustiz auch eine kriminaltechnische (spurentechnische) forensische Untersuchung der beschlagnahmten Gegenstände. Insbesondere müsse näher geklärt werden, inwieweit es sich um (aus privaten Sammlungen, Museen oder Kirchen) gestohlene Objekte handle bzw. um Fundstücke aus illegalen Raubgrabungen. Dass gegen die Ehefrau des Beschwerdeführers in der Schweiz eine separate Strafuntersuchung hängig sei, stehe der Rechtshilfe nicht entgegen.

6.4. Dass die italienischen Behörden eine Herausgabe der Gegenstände nicht nur zu Beweis- sondern auch zu Einziehungszwecken beantragt hätten, stellt kein Rechtshilfehindernis dar. In der Schlussverfügung sowie im angefochtenen Entscheid wird die Herausgabe ausdrücklich nur zu Beweiszwecken bewilligt. Die Rechtshilfevoraussetzungen von Art. 6 EUeR und Art. 74 IRSG sind grundsätzlich erfüllt. Zwar kann der ersuchte Staat die Übergabe von Gegenständen, um deren Übermittlung ersucht worden ist, aufschieben, wenn er sie selbst für ein anhängiges Strafverfahren benötigt (Art. 6 Ziff. 1 EUeR; Art. 74 Abs. 3 IRSG). Dass dies der Fall wäre, wird jedoch von den kantonalen Strafjustizbehörden mit sachlich nachvollziehbaren Argumenten verneint. [...]

Entgegen der Ansicht des Beschwerdeführers ergibt sich auch aus dem Urteil des Bundesgerichtes 1A.117/2000 vom 26. April 2000 (publiziert in SJ 2000 I S. 501 ff.) für den vorliegenden Fall kein Rechtshilfehindernis. Dort ging es um die Anfechtung einer Übertragung der Strafverfolgung an die italienischen Behörden (Art. 88-93 IRSG). Das Bundesgericht wies die gegen den Delegationsentscheid (und die auf Art. 90 IRSG gestützte Herausgabe von beschlagnahmten Gegenständen) erhobene Beschwerde der Betroffenen ab, soweit es darauf eintrat. Das Bundesgericht erwog, auch Art. 74a Abs. 4 lit. d IRSG bzw. Art. 74 Abs. 3 IRSG könnten der Herausgabe (gestützt auf Art. 90 IRSG) nicht mehr entgegenstehen, wenn das schweizerische Strafverfahren an den ersuchenden Staat zu delegieren ist (E. 2c). Daraus lässt sich keineswegs ableiten, dass in allen Fällen (wie dem vorliegenden), in denen keine Übertragung der Strafverfolgung an das Ausland erfolgt ist, 
eine Herausgabe zu Beweiszwecken nach Art. 74 IRSG ohne weiteres zu verweigern wäre.

6.5. Es fragt sich schließlich noch, ob der Beschwerdeführer als gutgläubige dritte Person im Sinne von Art. 74 Abs. 2 IRSG zu behandeln ist. Er selbst wird im italienischen Strafverfahren des illegalen Kulturgütertransfers sowie der Hehlerei, Nichtanmeldung von archäologischen Funden und Zugehörigkeit zu einer kriminellen Vereinigung angeschuldigt. Im in der Schweiz hängigen Strafverfahren gegen seine Ehefrau hat diese ihn bei Einvernahmen durch die Staatsanwaltschaft teilweise mitbelastet. Im Ersuchen und dessen Beilagen werden konkrete Verdachtsgründe gegen den Beschwerdeführer dargelegt (vgl. oben, E. 4.2). Sein guter Glaube (im Sinne von Art. 74 Abs. 2 IRSG) ist daher zu verneinen (vgl. zu dieser Praxis auch den bereits zitierten BGE 1A.117/2000, E. 2f).

6.6. Nach dem Gesagten ist die rechtshilfeweise Herausgabe zu Beweiszwecken hier grundsätzlich zulässig. Sie entspricht im Übrigen auch dem Sinn und Geist des UNESCO-Kulturgütertransfer-Abkommens (vgl. insbes. Art. 2 Ziff. 1, Art. 7 lit. b/ii, Art. 9 und Art. 13 des Abkommens). Die italienischen Behörden sind allerdings verpflichtet, die zu Beweiszwecken übermittelten Gegenstände so bald wie möglich an die schweizerischen Behörden zu retournieren (Art. 6 Ziff. 2 EUeR). Ein Verzicht auf die Rückgabe ist nicht erfolgt. Die kantonalen Behörden haben die Herausgabe von tausenden antiken Wertgegenständen verfügt. Dementsprechend sind hohe Vermögensinteressen der Betroffenen tangiert. Umso mehr muss der streitige Rechtshilfeentscheid klar, justiziabel und vollständig ausfallen. Im vorliegenden Fall ist (bei der Formulierung des Dispositivs sowie beim Rechtshilfe-Vollzug) daher besonderes Augenmerk darauf zu legen, dass Art. 74a IRSG nicht umgangen oder missachtet wird.

6.7. Im Dispositiv (Ziffer 2) der streitigen Schlussverfügung vom 22. Februar 2006 wird die Herausgabe verschiedener Unterlagen und Gegenstände angeordnet. Dem Dispositiv lässt sich nicht entnehmen, ob die Herausgabe zur definitiven strafrechtlichen Einziehung oder nur vorläufig zu Beweiszwecken erfolgt. Aus den Erwägungen der Schlussverfügung (Seite 3) ergibt sich immerhin, dass "die erhobenen Beweismittel in Anwendung von Art. 74 IRSG der ersuchenden Behörde herauszugeben" sind. In den Erwägungen des angefochtenen Entscheides wird dies bestätigt. Das Strafgericht hat jedoch keine Präzisierung des Dispositives der Schlussverfügung angeordnet. [...]

6.8. Die Vorkehren der kantonalen Behörden zur Sicherung der Rückgabe der zu Beweiszwecken herauszugebenden Vermögenswerte genügen im hier zu beurteilenden Fall den Anforderungen des EUeR und IRSG nicht. Das Dispositiv der Schlussverfügung ist in dem Sinne zu verdeutlichen und zu ergänzen, dass die rechtshilfeweise Herausgabe nur vorläufig zu Beweiszwecken erfolgt (Art. 6 Ziff. 2 EUeR i.V.m. Art. 74 IRSG). Eine weitere Verwendung der herausgegebenen Gegenstände für eine allfällige strafrechtliche Einziehung müsste von den italienischen Behörden separat beantragt und von den schwei- zerischen Rechtshilfebehörden ausdrücklich bewilligt werden. Ein entsprechendes förmliches Ersuchen müsste sich auf ein rechtskräftiges gerichtliches Einziehungsurteil stützen (vgl. BGE 131 II 169 E. 6 S. 175; 123 II 268 E. 4a S. 274, E. 4b/aa S. 275, 595 E. 4e-f S. 604-606, E. 5e S. 611 f.). Auch auf den entsprechenden Spezialitätsvorbehalt hat das BJ beim Vollzug der bewilligten Rechtshilfe ausdrücklich hinzuweisen.

Zwar ist der Beschwerdeführer nicht als gutgläubige Drittperson im Sinne von Art. 74 Abs. 2 IRSG zu betrachten. Im vorliegenden Fall drängt sich jedoch (in Nachachtung von Art. 6 Ziff. 2 EUeR) eine analoge Sicherung der Wertgegenstände im Vollzugsstadium auf. Vor dem Vollzug der Rechtshilfe muss das BJ von den italienischen Behörden daher die förmliche Zusicherung einholen, dass innert einer vom BJ anzusetzenden angemessenen Frist entweder eine kostenlose Rückgabe der Wertgegenstände an die schweizerischen Behörden erfolgt, oder aber ein neues Rechtshilfeersuchen, in dem Italien gestützt auf ein rechtskräftiges gerichtliches Einziehungsurteil die definitive Überlassung der herausgegebenen Gegenstände zu Einziehungszwecken beantragt.

Anzeichen, wonach die in diesem Sinne präzisierte und bewilligte Rechtshilfe für eine unzulässige strafrechtliche Einziehung missbraucht werden könnte bzw. dass die italienischen Behörden einen ausdrücklichen schweizerischen Spezialitätsvorbehalt missachten würden, bestehen nicht. Dabei ist auch dem Vertrauensgrundsatz im internationalen Rechtshilfeverkehr Rechnung zu tragen. [...]

\section{Demnach erkennt das Bundesgericht:}

1. Die Verwaltungsgerichtsbeschwerde wird im Sinne der Erwägungen teilweise gutgeheißen.

2. Das Dispositiv der Schlussverfügung der Staatsanwaltschaft Basel-Stadt vom 22. Februar 2006 wird (durch eine neue Ziffer 4) wie folgt ergänzt: Die Herausgabe erfolgt (im Sinne von Art. 6 Ziff. 2 EUeR und Art. 74 IRSG) nur vorläufig zu Beweiszwecken. Eine weitere Verwendung der herausgegebenen Gegenstände für eine allfällige strafrechtliche Einziehung müsste von den italienischen Behörden separat beantragt und von den schweizerischen Rechtshilfebehörden ausdrücklich bewilligt werden; ein entsprechendes förmliches Ersuchen müsste sich auf ein rechtskräftiges gerichtliches Einziehungsurteil stützen.

3. Der Vollzug der Rechtshilfe wird von der zusätzlichen Bedingung abhängig gemacht, dass die ersuchende Behörde folgende förmliche Zusicherung abgibt: Innert der vom Bundesamt für Justiz anzusetzenden Frist erfolgt entweder eine kostenlose Rückgabe der Wertgegenstände an die schweizerischen Behörden, oder aber ein neues Rechtshilfeersuchen, in dem die italienischen Behörden gestützt auf ein rechtskräftiges gerichtliches Einziehungsurteil die definitive Überlassung der herausgegebenen Gegenstände zu Einziehungszwecken beantragen.

4. Im Übrigen wird die Beschwerde abgewiesen, soweit auf sie eingetreten werden kann. 